\title{
VALIDATION OF AN ERYTHROCYTE SEDIMENTATION RATE ANALYZER WITH MODIFIED WESTERGREN METHOD
}

\author{
Asha Patil, Deepak Nayak M., Sushma V. Belurkar, SeemitrVerma
}
1. Assistant Professor, Department of Medical Laboratory Technology, Manipal College of Allied Health Sciences, Manipal University, Manipal.
2. Assistant Professor, Department of Pathology, Melaka Manipal Medical College, Manipal, Manipal University.
3. Associate Professor, Department of Pathology, Kasturba Medical College. Manipal, Manipal University.
4. Junior Resident, Department of Pathology, Kasturba Medical College. Manipal, Manipal University.

\author{
CORRESPONDING AUTHOR \\ Dr. Deepak Nayak. M, \\ Department of Pathology, \\ Melaka Manipal Medical College, \\ Manipal University,Manipal-576104. India \\ E-mail: deepzienator@gmail.com \\ Ph: 00919901920537
}

\begin{abstract}
VesMATIC cube80 $0^{\mathrm{TM}}$ is a newly developed automated method for measurement of erythrocyte sedimentation rate (ESR). AIMS: The aim of our study was to compare the ESR values by VesMATIC cube $80^{\mathrm{TM}}$ against the modified Westergren method. MATERIALS AND METHODS: This cross-sectional study was conducted at clinical laboratory in Kasturba Hospital, Manipal on 162 patients. The samples taken were as per the recommendations charted out by International Council for Standardization in Hematology (ICSH) for comparing automated and manual Westergren method. STATISTICAL ANALYSIS USED: Bland and Altman statistical analysis was applied for evaluating VesMATIC cube $80^{\mathrm{TM}}$ against the conventional Westergren method. RESULTS: The analysis revealed a low degree of agreement between the manual and automated methods especially for higher ESR values $(25 \mathrm{~mm} / \mathrm{hr}$.), i.e., mean difference -0.44 (95\% limits of agreement, -41.23 to 40.37). This discrepancy which is of clinical significance was less evident for ESR values in the normal range $<25 \mathrm{~mm} /$ hour $(-1.73$ mean of difference; -18.0 to 10 limits of agreement). CONCLUSIONS: The fully automated system VesMATIC cube80 ${ }^{\mathrm{TM}}$ for ESR measurement tends to underestimate the manual ESR readings. Further studies and validation experiments would be required.
\end{abstract}

KEYWORDS: erythrocyte sedimentation rate, VesMATIC cube80 ${ }^{\mathrm{TM}}$, Westergren method

INTRODUCTION: Erythrocyte sedimentation rate (ESR) is used as a parameter for prognostication and even diagnosis of certain clinical conditions. It is still widely used in clinical practice as an indicator of inflammation, infection, trauma, or malignant disease. Apart from complete blood counts (CBC) and peripheral smear, ESR is often preferred by clinicians in the requisition forms in almost all laboratories. The most satisfactory method of performing the test was introduced by Westergren in 1921. ${ }^{1}$ The original method recommended by International Council for Standardization in Hematology (ICSH) is based on that of Fahraeus and Westergren. ${ }^{1,2}$. Subsequently, modifications of this reference method were made and ICSH guidelines now allow for the use of alternative ESR techniques provided that comparability with the Westergren method is achieved. ${ }^{3}$ 
Over the last few years, newer and safer methods have evolved to determine ESR accurately without added risks. The VesMATIC cube $80^{\mathrm{TM}}$ analyzer is a new automated instrument for measuring ESR. The advantage conferred by this automated method is that it can generate the ESR readings in 30 minutes of 100 patients with all the temperature corrections at $18^{\circ} \mathrm{C}$. Additionally, the same EDTA sample collected for complete blood counts can also be used for ESR analysis by this automated machine.

However, there are no reports regarding the validity of ESR measurement using the VesMATIC cube $80^{\mathrm{TM}}$ analyzer.

AIMS AND OBJECTIVES: The study was conducted with the primary objective of comparing the VesMATIC cube80 ${ }^{\mathrm{TM}}$ analyzer with the modified Westergren method for the analysis of ESR.

MATERIALS AND METHODS: It was a cross-sectional study done on routine haemogram samples over a period of two months in April 2012. An approval from the Institutional Ethics Committee, Kasturba Hospital, Manipal was obtained before the study. A venous blood sample was obtained from 200 random patients who had a requisition form, marked for both CBC and ESR; arriving at the sample collection centre. All the samples were evaluated using the 2 methods in our clinical laboratory as per the methodology for each of the individual methods. All the ESR tests were carried out within 3 hours from the time of collection.

INCLUSION CRITERIA: Patients from both sexes and all age groups were included in the study. No controls were included in the study.

EXCLUSION CRITERIA: Blood collected by vein puncture taking more than 30 seconds and with excessive venous stasis were excluded from the study. Blood samples which were not in proper proportions to the anticoagulant, strongly lipemic, hyperbilirubinemic and hemolyzed samples were also excluded.

SAMPLES: Under all aseptic precautions, samples were collected from the antecubital vein using a $10-\mathrm{ml}$ syringe with $24 \mathrm{G}$ needle. Four milliliter of blood sample was drawn in the two special 2-ml EDTA vacutainers containing $1.5 \mathrm{mg} / \mathrm{ml}$ of EDTA and mixed immediately five times.

CONVENTIONAL WESTERGREN METHOD: In this method, a disposable, plastic tube with a bore size of $2.55 \mathrm{~mm}$ and a length of $230 \mathrm{~mm}$, vertically aligned, open at both ends was used. The pipette was filled with K3 EDTA anticoagulated venous blood to a height of at least $200 \mathrm{~mm}$. The sedimentation occurring at 60 minutes from the beginning of the test was noted in $\mathrm{mm}$ /hour equivalent to the Westergren ESR.

VESMATIC CUBE80 ${ }^{\mathrm{TM}}$ INSTRUMENT: READING PRINCIPLE: One hundred infrared barriers vertically cover 100 test tube positions. At $0.2 \mathrm{~mm}$ intervals, all 100 positions on the reading plate are analyzed at the same time. As soon as the reading plate comprising hundred pairs of infrared rays begins to rise, the indicating system intercepts any position occupied by samples containing the right level of blood. After approximately 3 minutes, the actual analysis begins. The computer records the zero time of each sample at regular intervals of 3 minutes for a total of 30 or 60 minutes. The instrument automatically converts the temperature to $18^{\circ} \mathrm{C}$ (Manley's normogram) and gives the reading in 30 minutes. 
STATISTICAL ANALYSIS: Statistical analysis was performed using SPSS 16.0 for Windows (SPSS Inc., Chicago, IL, USA). Evaluation of method was done as described by Bland and Altman. Modified Westergren method was considered as the reference method. Therefore mean value for ESR with both the methods is plotted against the difference between the Westergren and the VesMATIC cube $80^{\mathrm{Tm}}$. The $95 \%$ limits of agreement were calculated as $d \pm 1.96$ SD where $d=$ mean difference between the two measurements; and SD = standard deviation of differences.

RESULTS: Out of a total of 162 samples, 71 samples were within the reference range used in our hospital (0-25 mm/hour), while 91 samples had higher ESR values of more than $25 \mathrm{~mm} /$ hour. Agreement between the results obtained by manual and automated method for one hour is given below (Fig.1). The results obtained with the reference method (Modified Westergren) were plotted against the difference between the reference and the automated method. The mean difference between the two methods and 95\% limits of agreement at 1 hour was found to be -1.01 (95\% limits of agreement are from 63 to -44$)$. Thus we estimate that the 1 hour ESR readings for $95 \%$ of subjects as measured by this automated method will be 44 $\mathrm{mm}$ /hour below the manual method or $63 \mathrm{~mm} /$ hour above it. This was unacceptable for clinical interpretation since there was a marked discrepancy between the reference and the automated methods. This variation was particularly evident for samples with high ESR readings greater than $25 \mathrm{~mm}$ /hour. Hence for samples with high manual ESR values, the mean difference was estimated to be -0.44 (95\% limits of agreement: -41.23 to 40.37 ). This was also markedly different from the corresponding values for ESR values less than $25 \mathrm{~mm} /$ hour i.e. mean difference was -1.73 (95\% limits of agreement: -18 to 10$)$.Thus our inference was that the samples with high ESR values vary considerably around the mean difference compared with samples which had normal ESR readings.

DISCUSSION: Erythrocyte sedimentation rate continues to be used as an indicator of inflammation and infection in all levels of laboratory services; despite being a non-specific test. The gold standard technique for measuring ESR is the Westergren method. However, this method has distinct disadvantages which limit its applications. ${ }^{4}$ Over the years, several attempts to introduce automated systems for measuring ESR have evolved. The modifications introduced include the use of unopened blood collection tubes, vacuum-controlled aspiration of the sample (which intends to provide a correct dilution with the anticoagulant) and automated mixing.5-8 Due to the rise in the prevalence of diseases such as hepatitis B and HIV, transmitted by blood, safety precautions against contamination of laboratory personnel are imperative. Using an automatic analyzer such as VesMATIC cube $80^{\mathrm{TM}}$ is one evolutionary step in this regard.

Several automated systems have been introduced since the last two decades and have been evaluated for performance with each other as well as with the standard Westergren method. Few eminent examples include VesMATIC 60 (Menarini Diagnostics, Milan, Italy), Sediscan (Becton Dickinson, France) among many others. These automated techniques offer more benefits in terms of reduced biohazard risks, speedy processing time, and quicker results. However, it is an absolute necessity to validate these instruments against the standard Westergren method to enable routine use and also to substitute the standard ESR method.

VesMATIC cube $80^{\mathrm{TM}}$ is a newly developed bench top automated method which can give the ESR readings in 30 minutes (equivalent to 1 hour Westergren) of 100 patients with all the temperature corrections at $18^{\circ} \mathrm{C}$ using infrared barriers which are not seen with the usual standardized methods for ESR. The added advantage of VesMATIC cube80 ${ }^{\mathrm{TM}}$ is that there is no 
external influence on the final reading such as temperature, contaminating dust particles, tilting of tube, and ratio of diluents. The number of samples that can be processed with this method is higher (maximum of 100 samples can be processed at a time) than the manual method; which is a boon in any tertiary care hospital with a heavy workload. It also has additional benefit such as usage of a single sample for both CBC and ESR, thus preventing excess sample collection.

In the present study, the results obtained with the VesMATIC cube $80^{\mathrm{TM}}$ analyzer were compared with the modified Westergren method using the agreement analysis of Bland and Altman. ${ }^{9}$. Agreement analysis is a more sensitive method than the correlation coefficient for comparison between the two methods. The Bland and Altman analysis revealed marked discrepancy between the two methods especially for values on the higher side. The mean of the difference between the two methods was found to be -1.01 (95\% limits of agreement are from 63 to -44 ) for 1 hour readings. This finding was clinically significant and hence unacceptable due to marked discrepancy in the readings between the two methods. This discrepancy was not evident for normal ESR values less than $25 \mathrm{~mm}$ /hour (mean of difference -1.73 ; $95 \%$ limits of agreement: -18 to 10$)$.

When we referred to the available literature, we found that such discrepancies with the ESR automated analyzers have also been demonstrated previously by various authors.10,11 while some authors like Caswellet al ${ }^{11}$ have showed low agreement values with the Westergren ESR; other shave showed a good correlation and agreement results with the same method.12 In a similar analysis of the fully automated system, Alfadhli et al showed low-agreement results with the higher ESR values and comparable results in the normal ESR range. ${ }^{13}$ More recently, newer automated systems for measuring ESR have shown comparably good agreement results enabling their use in clinical laboratories with a high workload as well as for emergency laboratories. ${ }^{14,15}$ Our study results concurred with the some of the above.

The use of Bland and Altman analysis for evaluating the agreement between the two methods not only assesses the mean of the difference $(d)$ between the two methods (i.e., bias) but also the limits of agreement by calculating the standard deviation of the differences $(d \pm$ $2 \mathrm{SD})$. Only when the difference $(d \pm 2 \mathrm{SD})$ does not affect the clinical interpretation, the two methods can be used interchangeably. In other words, the probability of obtaining similar results with the two methods is doubtful; hence the difference in the results obtained with both the methods should be clinically acceptable. Only then, one can justify the new method replacing the standard method in the hospital.

CONCLUSION: On comparing the VesMATIC cube80 ${ }^{\mathrm{TM}}$ analyzer with the modified Westergren method for the analysis of ESR, a marked discrepancy was noted for high ESR values. However, this was not evident for normal ESR values. Thus we deduce that the automated system tended to underestimate the manual readings for ESR values on the higher range which is clinically unacceptable. Further validation experiments and studies, including a possible correction factor would be required to achieve complete reliability on the automated analyzer.

\section{REFERENCES:}

1. Westergren A. Studies of the suspension stability of the blood in pulmonary tuberculosis. Acta Med Scand 1921;54:247-282.

2. Fahraeus R. The suspension stability of blood. Acta Med Scand 1921;55:1-228. 
3. International council for Standardization in hematology. ICSH recommendation for measurement of ESR. J Clin Pathol 1993; 46:198-203.

4. Atas A, Cakmak A, Soran M, Karazeybek H. Comparitive study between the Ves-Matic and Micro erythrocyte sedimentation rate method. J Clin Lab Anal 2008; 22:70-72.

5. Patton WN, Meyer PJ, Stuart J. Evaluation of sealed vacuum extraction method (Seditainer) for measurement of erythrocyte sedimentation rate. J Clin Pathol 1989; 42:313-317.

6. Besson I, Kinder M, Jou JM, VivesCorron JL. The evaluation of three automatic systems for determining the velocity of globular sedimentation. Blood 1995; 40:103-107.

7. Plebani M, De Toni S, Sanzari MC, Bernardi D, Stockreiter E, et al. The TEST1 automated system: A new method for measuring the erythrocyte sedimentation rate. Am J Clin Pathol 1998; 110:334-340.

8. Fernandez de Castro M, Fernandez Calle P, Viloria A, Larrocha C, Jimenez MC. Evaluation of a fully automated system for determination of the erythrocyte sedimentation rate. Blood 1989; 34:1-9.

9. Bland JM, Altman DG. Statistical method for assessing agreement between two methods of clinical measurement. Lancet 1986; 1:307-310.

10. Plebani M, De Toni S, Sanzari MC, Bernardi D, Stockreiter E, et al. The TEST1 automated system: A new method for measuring the erythrocyte sedimentation rate. Am J Clin Pathol 1998; 110:334-340.

11. Caswell M, Stuart J. Assessment of Diesse Ves-matic 20 automated system for measuring erythrocyte sedimentation rate. J Clin Pathol 1991; 44:946-949.

12. Fares AK. Evaluation of the Ves-matic 20 -An automated system for the determination of the erythrocyte sedimentation rate. Middle East Laboratory 2001; 4:19-23.

13. AlFadhli SM, Al-Awadhi AM. Comparison of erythrocyte sedimentation rate measurement by the automated SEDI system and conventional Westergren method using the Bland and Altman statistical method. Med PrincPract 2005; 14:241-244.

14. Arikan S, Akalin N. Comparison of the erythrocyte sedimentation rate measured by the Micro Test 1 sedimentation analyzer and the conventional Westergren method. Ann Saudi Med 2007;27:362-5.

15. Mahlangu JN, Davids M. Three-way comparison of methods for the measurement of the erythrocyte sedimentation rate. J Clin Lab Anal 2008;22:346-52. 


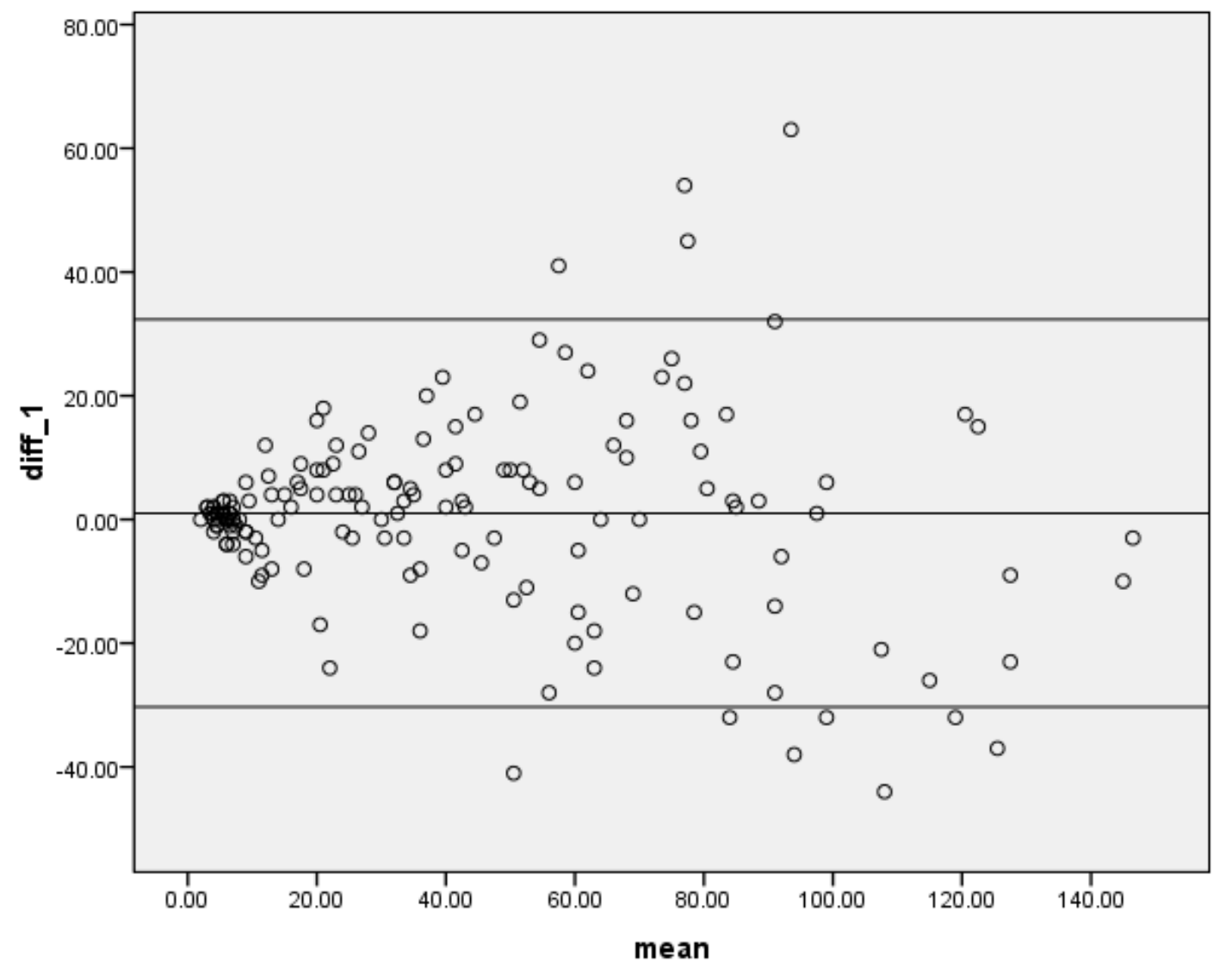

Fig.1: Bland and Altman analysis of the comparison between reference and automated method at 1 hour, mean difference -1.01 (95\% limits of agreement are from 63 to -44 ). 